\title{
The Obs-Services and their potentials for biodiversity data assessments with a test of the current reliability of photo-identification of Coleoptera in the field
}

\author{
Christian Molls
}

\begin{abstract}
The current reliability of species identifications by the Nature Identification API (NIA) of the app ObsIdentify is tested with a Coleoptera (Insecta) sample set from Germany. Seventy-five photographic beetle records taken with a smartphone camera under "average user" conditions are analysed in terms of correctness of the app's identification result on various taxonomic levels, the displayed confidence level of the identification and the time until validation of the results. More than $60 \%$ of samples were identified correctly at the species level, but only $53 \%$ were validated within a month. The mechanisms by which users can upload pictures of their observations to be identified by the artificial intelligence and the validation process by experts are briefly explained. Regional specifics and further opportunities for data usage as well as currently existing problems are discussed and improvements are suggested. The expert validation of records is identified as a huge quality advantage of the Obs-Services. They are generally found to be a promising tool for lay people and professional institutions, despite still existing deficiencies such as identification failure in mutilated specimens, cryptic and rare species, doubtful species rarity ratings as well as the still insufficient capacity of validation. Experts and institutions are encouraged to volunteer as validators and collaborators.

Keywords: artificial intelligence, beetle, citizen science, deep learning, image recognition, nature identification, API observation

Christian Molls, RWTH Aachen University, Institute for Environmental Research, Worringerweg 1, D-52074 Aachen, Germany. christian.molls@rwth-aachen.de
\end{abstract}

\section{Introduction}

Digitization and the use of artificial intelligence (AI) have become driving factors for technological innovation, including biodiversity research. Deep-learning technologies (algorithm-based machine-learning methods) have significantly improved during the last decade (LeCun et al. 2015). Similarly, digital photography opportunities have also massively improved. Automated wildlife identification techniques have been developed and tested for camera trap pictures (e.g., Gomez Villa et al. 2017), marine benthos samples (e.g., Beijbom et al. 2015), plants (e.g., Oncevay-Marcos et al. 2015), and many more scientific approaches. In the past few years, several smartphone-operated applications for the automatic identification of a large variety of organisms were launched (e.g., https://observation.org/apps/obsidentify/, https://www.inaturalist.org/pages/seek_app) that allow the use of AI for taxonomic identifications even by laypeople.

With the photo-based identification app ObsIdentify (https://observation.org/apps/obsidentify/), launched in 2018 by the foundation Observation International, it is possible for citizen scientists as well as professionals to document and upload data on practically any group of organisms they find in 
the field on a global scale. The software uses a deeplearning image recognition algorithm called Nature Identification API (Naturalis Biodiversity Center, Leiden, The Netherlands and COSMONiO Imaging BV, Groningen, The Netherlands). The observations are then publicly accessible on the website observation.org, making it a kind of digital museum to which anyone can contribute. With the app, especially younger people might become interested in participating in biodiversity data gathering, since all they need to do is take a picture with their smartphone. The app rewards the users with badges when they have found a certain number of species of a group which adds motivation to "fill the Pokédex".

Such digitized data can help updating distribution maps of species which are often outdated and can nowadays change rapidly due to climate change and other impacts on the habitats. Hochkirch et al. (2020) promoted the use of apps like ObsIdentify in their strategy to address data deficiency in neglected biodiversity in the next decade. Roger et al. (2020) comprehensively summarized the recent growth in citizen science and its benefits but also its challenges.

Kaufmann \& Lindner (2021) tested several species identification apps based on certain criteria including data quality and accessibility, covered taxa and regions. They concluded that ObsIdentify matches those criteria best. Partnerships and implementations of the Obs-Services (website and apps) in Haus der Natur, Salzburg; Naturalis Biodiversity Center, Leiden; LWL Museum für Naturkunde, Münster;
Sociedad Española para la Conservación y Estudio de los Mamíferos (SECEM), Spain; and Deutsche Gesellschaft für Herpetologie und Terrarienkunde (DGHT) (Schlüpmann et al. 2021) have already proven their usefulness and scientific compatibility (Observation International 2021).

ObsIdentify uses an image recognition algorithm that identifies species from geo-tagged photographs. It is trained with validated pictures on a regular basis. Within seconds upon cropping and uploading the picture of a specimen, the software suggests one or multiple results, usually at species level, with a confidence ranging from 0 to $100 \%$ (Fig. 1). The users can check associated taxonomic information and previously uploaded pictures of the suggested species and then pick the one they think is correct and save the observation. Location, time, and day are added automatically from the photo's specifications and are obligatory to submit an observation.

Photos can also be uploaded via the website https://observation.org/ or the app ObsMapp which both use the image recognition software. These two options provide opportunities to add more details to the observation as for instance, number and sex ratio of individuals, counting method, stage of development, associated substrate etc. which increase the value of the observation. Additionally, the user can type the species name manually and does not have to choose from the suggested results of the algorithm which not always include the correct species. Location, time, and date of the observation can

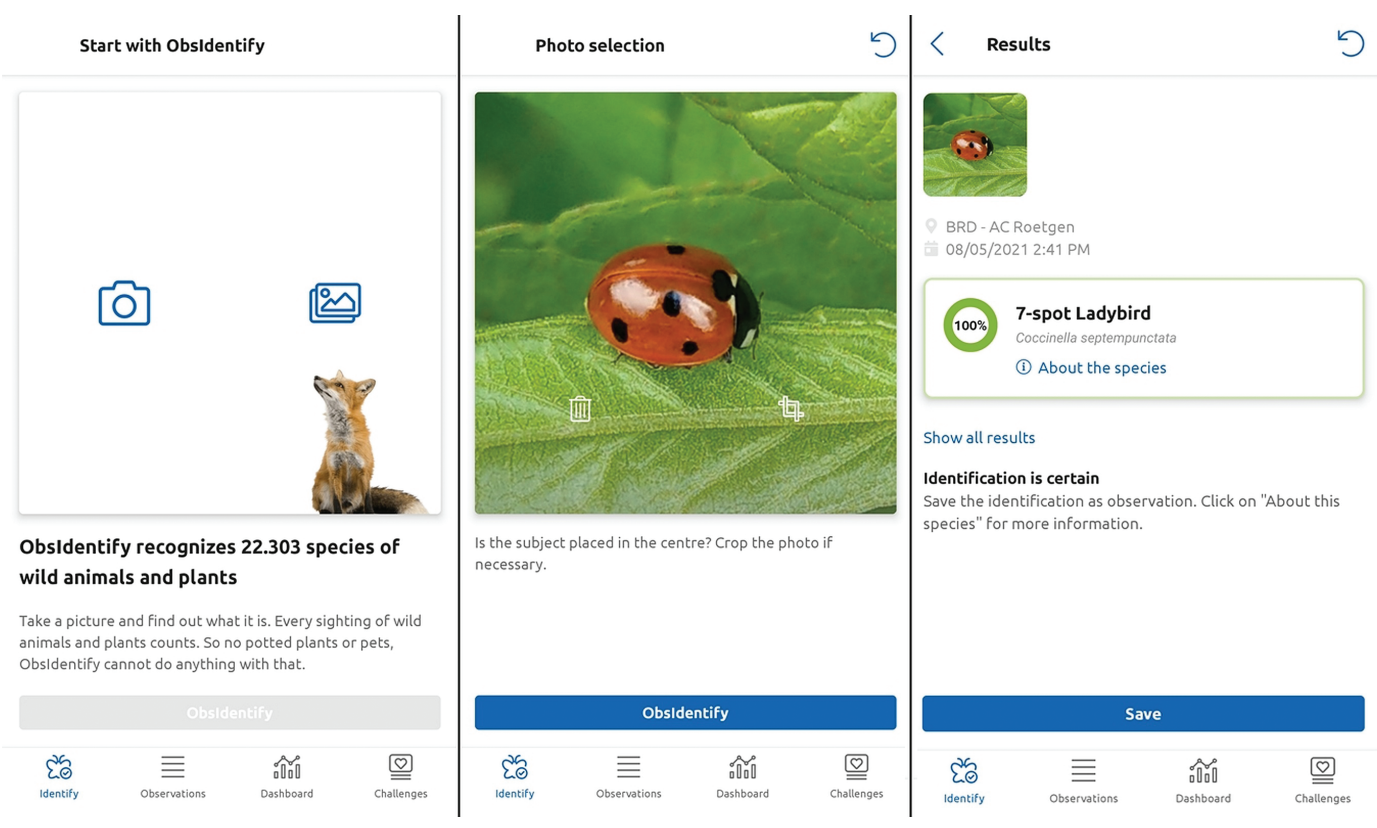

Fig. 1. Layouts during different steps of submission in the app ObsIdentify. 
also be manually added in these two platforms, if the photo's specifications do not include these data already. These additional options are not yet available in ObsIdentify. The main intention is to serve as a user-friendly application for lay people who quickly want to identify their observations "on the go".

A major advantage of the Obs-Services is a strict quality control by contracted but volunteering expert validators, who are specialized in a specific taxonomic group. In order to get accepted as a record, the uploaded observations must be verified by an authorized validator. Exceptions of automated validations are only applied to species that can practically not be misidentified and have been validated in the location before. New validators are usually suggested by other validators to the Board of Directors of the foundation "Observation International". In some cases, experts also lend themselves to the board, or they are actively enlisted. When their expertise is well proven, the board initiates a discussion with other validators, e.g. through the platform's online forum (a section of it, which is not open for general users). If no objections are raised and the validator candidates can prove their active observer/user status demonstrating enough familiarity with the platform's tools, new validators are contracted. They are not only assigned to taxonomic groups but also to geographical regions. However, a user who is a validator cannot validate his own records and still depends on other validators to confirm any submission. Validators can be ranked into "taxonomist" status, enabling them to add, delete or edit species names and properties (e.g., rarity rank, automated validation) in the database.

So far, the Netherlands, Belgium and Germany have most validators assigned whereas other regions, especially non-European countries, are underrepresented and validation progress is slow or even impossible there. Consequently, the app works most comprehensively in the former countries. When an observation was corrected, altered, validated, or commented on, users receive an e-mail, if they have not turned off this function. Users can contact administrators in order to add species that are not listed in the database and therefore could not be saved under the right name yet. Most species in Europe are covered but the species lists for other regions are still fragmentary. However, in principle there are no limitations to extending the coverage to any region of the world.

Once data are uploaded, they are made publicly accessible, if the user has not blocked this function. This accounts for validated and non-validated observations. Validated observations are used to train the deep-learning algorithm and they are regularly shared with the Global Biodiversity Information Facility (GBIF). All users can search for practically any observation uploaded to the database. A wide array of filters allows for precise searches, e.g., for a specific taxonomic group, time, or area. Exact locations of endangered, particularly sensitive or rare species are either automatically obscured or can be obscured by users and validators (in a radius up to $1000 \mathrm{~m}$ from the original location). This helps protecting these species from collectors and photo-tourists, which can have a severe impact on charismatic taxa, such as orchids and their habitats.

The current global trend of biodiversity loss is obvious but data on distributions and abundances are mostly very scarce for many megadiverse taxa like Coleoptera (Hochkirch et al. 2020). This is due to underfunding of taxonomic research and the fact that for many groups only a handful of experts are left who can identify species confidently (Engel et al. 2021).

In this paper, the current reliability of in-app results is tested for the first time on beetles (Coleoptera), which are a very diverse and the most species-rich order of animals. Coleoptera include many species similar in morphological appearance (including cryptic species), making it hard to identify them even for experienced coleopterologists. The author is an official validator with taxonomist status on observation.org for Coleoptera.

\section{Material and methods}

During two field excursions in and around DessauRoßlau, Germany, which is embedded in the UNESCO "Flusslandschaft Elbe Biosphere Reserve", photos of different species of Coleoptera were taken in their habitat with a smartphone (HTC U11; High Tech Computer Corporation, Taoyuan, Taiwan) equipped with a 12-megapixels camera. Photo samples were taken randomly during walks at different times and various places in the area. Pictures were generally taken quickly on the go with default camera settings, as probably done by an average ObsIdentify user. This means some details needed for identification might not be as obvious as in high-quality macro-photographs or may not be visible at all due to low zoom levels, blurriness or unsuitable angles. As most users would do, generally only one picture, depicting the complete (usually dorsal) habitus of a specimen, was uploaded for each sample. In few cases, dead and even mutilated specimens encountered during the excursions were photographed through a battery-illuminated Novex P-10 stereomicroscope (EUROMEX, Arnhem, The Netherlands). This included some small or hardly identifiable species. The images were loaded post-hoc into ObsIdentify (Version 2.0, downloaded on 10 May 2020) and cropped in-app in order to place the specimen in the centre of the image as required by the app (Fig. 2).

The top identification result and the respective in-app confidences were recorded (Fig. 3, Table 1). If the top in-app result was correct (based on the 


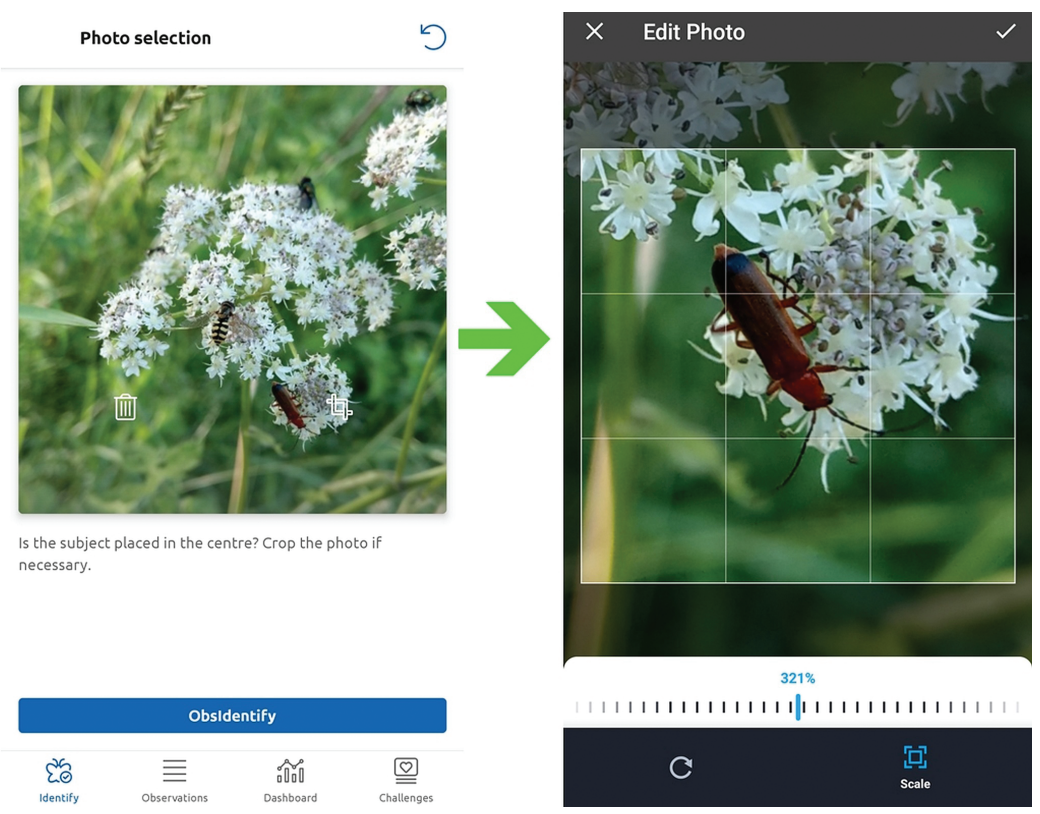

Fig. 2. For optimal results the specimen should be placed in the centre of the picture. This is possible via an in-app function.

- Correct species
- Error at species level
- Error at genus level
- Error at family level
- Error at higher taxonomic level

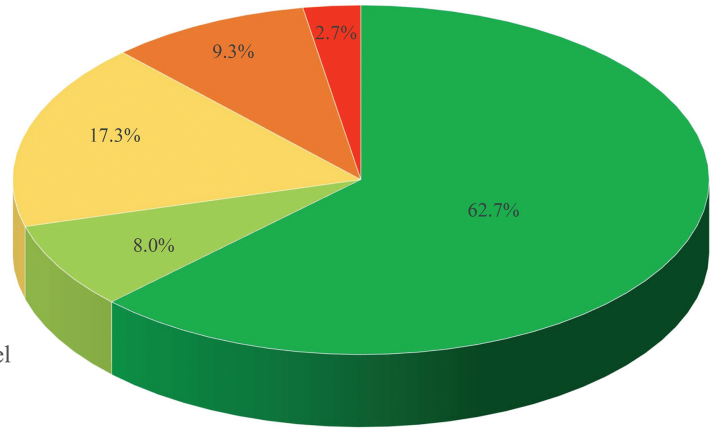

Fig. 3. Accuracy of top in-app results. Error at species level meaning the app identified the correct genus but not the correct species or only the genus was suggested.

author's expertise), it was uploaded via the app. In case the top result was incorrect, and the correct species was not included in the lower-confidence results (Table 2), the identification was manually corrected by the author via the website. This control identification was generally based on the author's expertise. Some specimens in question were identified with the help of keys on the website www.coleonet.de.

Time of upload and time of validation were also recorded (Table 3 ). A total of 75 specimens belonging to 71 species from 24 families were processed in this way. At the time of data gathering, the area of focus was not yet officially supported due to lack of assigned validators. The "location unknown" notifications in many of the figures shown here result from re-downloaded images which did not include the original image specifications. Records on correctness and confidence of the initial in-app identifications were taken from the original images that included the location. Some other tests with pictures unrelated to the excursions were performed, but those were not included in the statistical analysis. 
Table 1. In-app confidences of top result; sorted by correct and incorrect identifications.

\begin{tabular}{lcc}
\hline $\begin{array}{l}\text { Confidence of top } \\
\text { result (\%) }\end{array}$ & $\begin{array}{c}\text { Number of } \\
\text { results }\end{array}$ & $\begin{array}{c}\text { Percentage of uploads } \\
(n=75=100 \%)\end{array}$ \\
\hline $90-100$ (correct ID) & 43 & 57.3 \\
$75-89$ (correct ID) & 3 & 4 \\
$50-74$ (correct ID) & 1 & 1.3 \\
$<50$ (correct ID) & 2 & 2.7 \\
$90-100$ (incorrect ID) & 8 & 10.7 \\
$75-89$ (incorrect ID) & 4 & 5.3 \\
$50-74$ (incorrect ID) & 6 & 8 \\
$<50$ (incorrect ID) & 8 & 10.7 \\
Total & 75 & 100 \\
\hline
\end{tabular}

Table 2. Number of corrected identifications suggested in lower-confidence results.

\begin{tabular}{lcc}
\hline $\begin{array}{l}\text { Corrected ID included } \\
\text { in lower-confidence } \\
\text { results }\end{array}$ & $\begin{array}{c}\text { Number } \\
\text { of results }\end{array}$ & $\begin{array}{c}\text { Percentage of } \\
\text { corrected IDs } \\
(n=27=100 \%)\end{array}$ \\
\hline Yes & 6 & 22.2 \\
No & 21 & 77.8 \\
Total & 27 & 100 \\
\hline
\end{tabular}

\section{Results}

Out of 75 samples (Supplement 1; available at doi: $10.6084 / \mathrm{m} 9$.figshare.16866181), the app was able to identify 47 species correctly and listed them as top result accounting for $62.7 \%$ of all samples (Fig. $3)$. In six cases $(8.0 \%)$, the species was not correctly identified but as a species from the correct genus or as "correct genus spec." (a collective, but not specified identification for several representatives of a genus) was suggested as top result. This includes two samples which the author also could only confidently identify to genus level (Byrrhus spec., Nebria spec.) based on the photographs. For 13 specimens (17.3\%), the top result displayed an error at genus level but suggested a species belonging to the correct family. Another seven top results $(9.3 \%)$ suggested species from the correct order but were erroneous at family level. For remains of dead stag beetles (Lucanus cervus), the app suggested two misleading top results of a completely different class of Animalia, accounting for $2.7 \%$ of the data.

Of the 49 results listed as correct (Table 1), 43, accounting for $57.3 \%$ of all samples, had an inapp confidence of $90-100 \%$. Eight results (10.7\%) identified an incorrect species with a confidence of 90-100\%. At this confidence level the user must actively click the option "show more results" in order to see other suggestions. Below this confidence, multiple suggestions are listed automatically. In the confidence range of $75-89 \%$, three results $(4.0 \%)$
Table 3. Validation status of submitted observations.

\begin{tabular}{lcc}
\hline Validation status & $\begin{array}{c}\text { Number } \\
\text { of results }\end{array}$ & $\begin{array}{c}\text { Percentage of uploads } \\
(n=75=100 \%)\end{array}$ \\
\hline Within approx. 7 days & 38 & 50.7 \\
$\begin{array}{l}\text { Within approx. 1 } \\
\quad \text { month }\end{array}$ & 2 & 2.7 \\
Pending & 35 & 46.7 \\
Total & 75 & 100 \\
\hline
\end{tabular}

were correct; the result Byrrhus pilula was included here though the author is only confident at genus level. Four results $(5.3 \%)$ in this range of confidence were incorrect. In the range between 50 and $74 \%$ confidence, only one result (1.3\%) was correct, and six $(8.0 \%)$ were incorrect. Two results $(2.7 \%)$ were correct but had a confidence below $50 \%$, including the result Nebria spec. which the author could also not identify up to species level. In this range, eight results $(10.7 \%)$ were incorrect (Table 1$)$.

If the initial top result was incorrect, the correct identification was searched for in the list of suggestions with lower confidences. For a total of 27 samples, a correction had to be applied to the top result. Only in six cases was the correct identification listed within the lower-confidence results, accounting for $22.2 \%$ of total corrections. Twenty-one wrong identifications $(77.8 \%)$ had to be corrected by typing the species name manually on the website, as the correct name was not listed within the lower-confidence results of the algorithm (Table 2). The result Byrrhus pilula was included here as the author could only confidently confirm this result to genus level and Byrrhus spec. was not listed by the app.

Of the 75 submitted identifications, 38 (50.7\%) were validated within roughly seven days (Table $3)$. Two submissions $(2.7 \%)$ were validated within roughly a month and $35(46.7 \%)$ had their validation pending at the time of data acquisition (12 July 2021), with one validation in progress, meaning a validator was actively working on this submission.

Several pictures were used to test in how far the result is affected by the angle at which the specimen was photographed, or which zoom level was applied. An example is given in which the zoom level and centre placement were slightly altered several times and individually submitted (Fig. 4). The results varied in confidence and in suggested species.

On a few occasions, it was investigated if the background might influence species identification. On the tested photographs the correct species was correctly identified with high confidence, even on shape-dissolving backgrounds if the image was not too blurry (Figs 5, 6). 


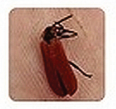

ERO- Oessou (Kreisfreie Shadt) 13.05.2021 14:20

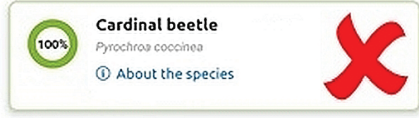

Show all results

Identification is certain

No location in the photo. Possibly the photo was made outside the geographical area of this identification service. Identification can be inaccurate.

Click on "About this species" for more information.

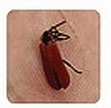

ERD - Oessau (Kreisfreie Stady) 13.05.2021 14:20

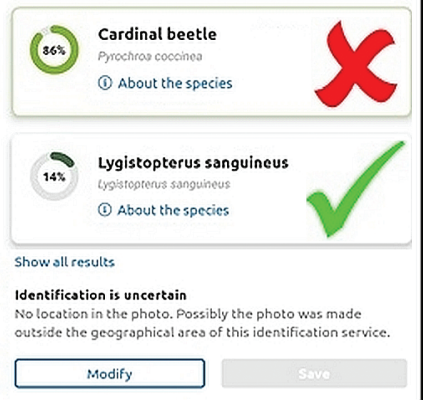

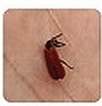

ERD - Dessau (Kreisfreie Stadt)

13.06.2021 14:20

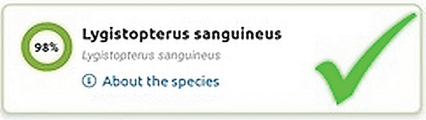

Show all results

Identification is certsin

No location in the ghoto. Possibly the photo was made outside the geographical area of this identification service. Identification can be inaccurate.

Click on "About this species" for more information.

Fig. 4. Different zoom levels and centre placements of the same picture bring different results. On the left 100\% confidence for Pyrochroa coccinea (error at family level). In the middle $86 \%$ confidence for P. coccinea and $14 \%$ for Lygistopterus sanguineus (correct species). On the right 98\% confidence for L. sanguineus.
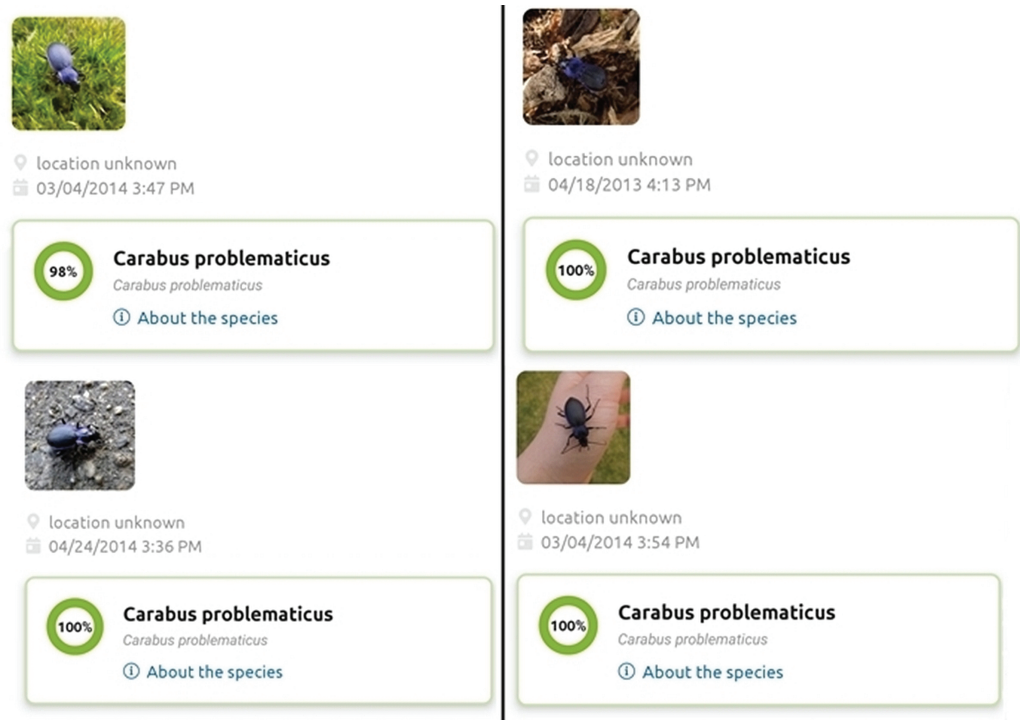

Fig. 5. Correct high confidence identification of Carabus problematicus on different backgrounds.

\section{Discussion}

Nearly two thirds of all submissions of beetle photographs were identified correctly in the current training state of the deep-learning image recognition algorithm. This is far from a perfect score but still a promising result considering that the correctly identified species were usually in the high-end confidence ranges and the algorithm is continuously trained for further improvement. As the pictures were of "average" quality and only one perspective was submitted for each photo, the results are quite pleasing. From the author's experience, good results are achieved when the length of the specimen is about half the length or width of the frame. However, correct and incorrect results can occur regardless of the angle, placement in the centre, zoom level, or total frame coverage.

Unfortunately, the majority of initially incorrect identifications by the app did not display the correct species name in lower-confidence results, sometimes not even suggesting other species automatically, because the wrong identification had a confidence of $90 \%$ or higher. This is a serious concern as lay people might tend to choose the top result and would rarely look up the provided species information page or 


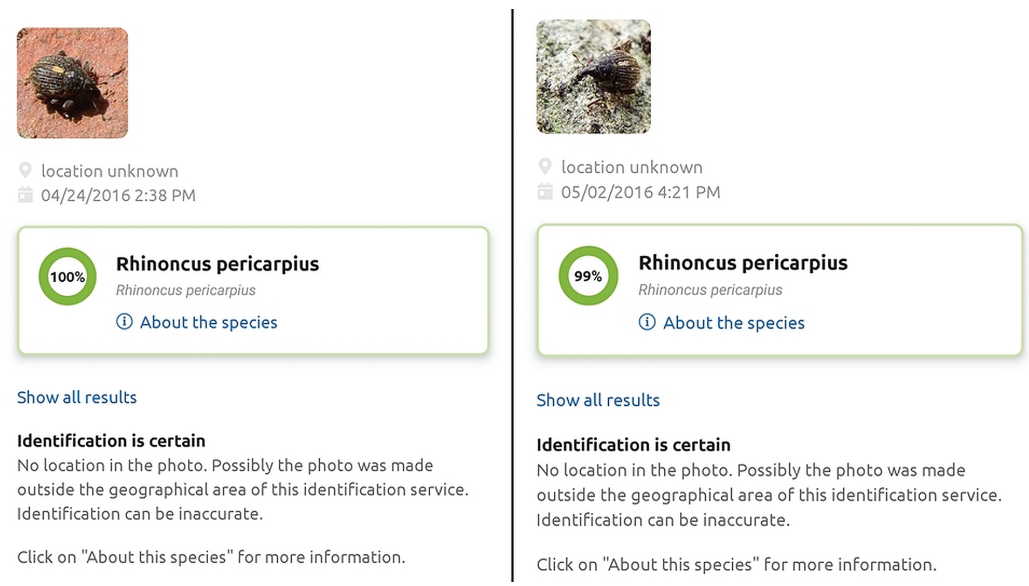

Fig. 6. Correct high confidence identification of Rhinoncus pericarpius on different backgrounds.
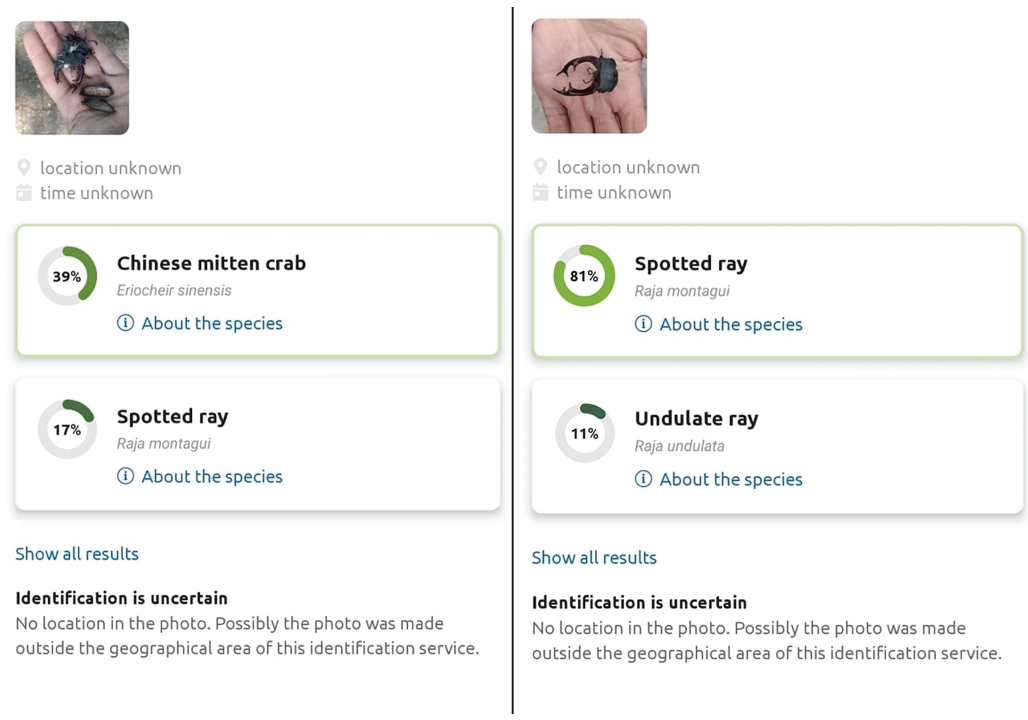

Fig. 7. Errors at class-level resulting from body fragments of Lucanus cervus leading to identification results of Eriocheir sinensis (Decapoda) and Raja montagui (Chondrichthyes).

other images of the suggested result for comparison with their own observation.

Externally easily identifiable species with a rather obvious lifestyle, like pollinators, generally lead to higher observation rates. Thus, they are more frequently correctly identified, for instance species from the families Cerambycidae and Coccinellidae. On the other hand, species that are morphologically somewhat cryptic and/or exhibit a more hidden lifestyle tend to be identified incorrectly, for example species from the families Curculionidae and Elateridae.

In most cases of misidentification, the results still led to the correct family at least. This is important as validators are mostly specialized on a certain family and usually only validate submissions that were initially uploaded in their section. But if an observation with an error higher than family level was submitted then chances are high that these submissions will not get corrected/validated.

Only submissions of specimen fragments $(n=2)$ resulted in an error higher than order level (Fig. 7), while the submissions of intact specimens of the same species (Lucanus cervus) usually lead to a correct high-end confidence result, as the author can confirm from previous submissions and his work as validator.

Users should generally be very critical with identification results from any specimen that is only 
seen in part. This applies even for well-documented taxa, as personally observed for photographs where specimens are partly covered by vegetation or other structures. For example, Lacerta agilis (Squamata) was identified as Proserpinus proserpina (Lepidoptera), probably due to similar patterns of the female lizard and the caterpillar. It should be kept in mind that characters that appear diagnostic for a human observer are not necessarily identical with patterns the AI recognizes.

Another serious issue is that some species listed as critically endangered and protected by law in Germany $($ Red List $=\mathrm{RL})$ and/or the EU [Habitats directive $=$ FFH (Fauna Flora Habitat)] were neither recognized as the top result nor suggested in the list of results. This means that these valuable observations may remain unrecognized until checked by a validator and the location is likely not to be obscured, which would be important for species of such status. In this data set this occurred for Cerambyx cerdo (RL 1, FFH II, IV), Protaetia speciosissima (RL 1), Lucanus cervus (RL 2, FFH II) and Lacon querceus (RL 1) (Fig. 8; Supplement 1 at doi: 10.6084/ $\mathrm{m} 9$.figshare.16866181). The first three of these represent "trophy" species sought after by collectors.

On observation.org, species are ranked by rarity indicated by icons of different shapes and colours. Many species, not only in Coleoptera, are currently ranked incorrectly in rarity. Rare and endangered species are often ranked as common, even charismatic species such as the yellow-bellied toad (Bombina variegata) which is critically endangered in most parts of its range and whose populations are still declining in recent years (Laufer et al. 2020). The same accounts for the afore-mentioned Cerambyx cerdo.
Thus, rarity ranks must be re-evaluated. Administrators with taxonomist status should make more use of the opportunity to alter the rarity rank of a species. Yet there are not enough administrators with taxonomist status especially for the megadiverse groups who could possibly assess all rarities in short time. Rarity ranks can and should be subdivided by the respective regions and observations of endangered taxa should be obscured by default. The latter is already the case for some taxa, e.g., orchids.

At the date of data consolidation for this paper (12 July 2021) $50.7 \%$ of submitted observations were validated within roughly seven days. This window of time is still in the range where people might remember details of the observation that might have not been submitted initially, but which are needed for validation. This may no longer be the case for observations validated after several weeks or months. Additionally, validators are less likely to validate older submissions as new submissions are shown first on the validation pages and older observations are, if at all, only occasionally worked on. Given that about half of the records in this data set were not validated even after several months, the need for more active validators is evident, especially for megadiverse and common taxa like Coleoptera.

In other taxonomic groups like vascular plants, birds, or Lepidoptera, as far as the author can tell from his submissions, the process of validation tends to be quicker and is more frequently within the seven-days period. Also, for Lepidoptera the identification results are generally of higher accuracy and with higher confidences compared to Coleoptera, probably due to their better AI-recognizable shapes and patterns and the better detection of individuals in

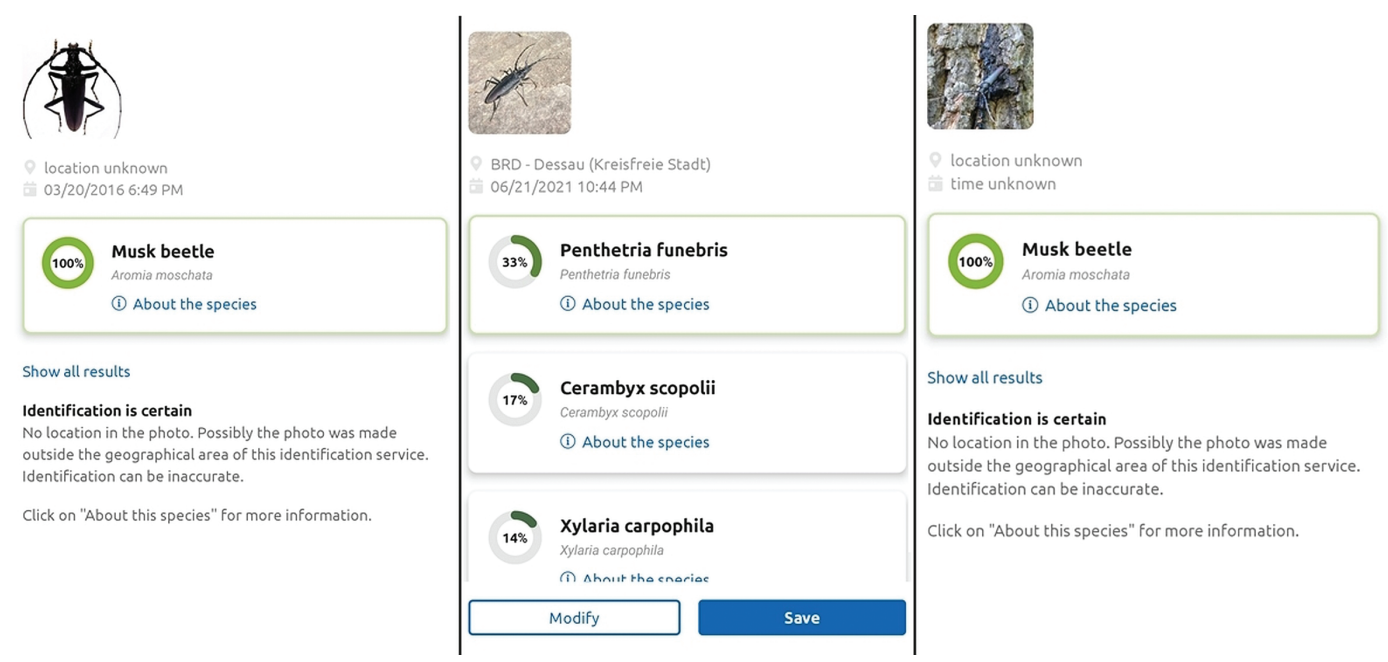

Fig. 8. The critically endangered and quite charismatic Cerambyx cerdo was misidentified in all three submissions for this species. 
the field, leading to a better current training level of the algorithm for this group. Lepidoptera receive significantly higher attention as being reflected by more submissions (947 432 in Lepidoptera vs. 127341 in Coleoptera) (https://old.observation.org/statistiek. php, 10 October 2021) and more validators (60 in Lepidoptera vs 28 in Coleoptera) (https://old.observation.org/users_rollen.php, 10 September 2021) which also leads to a better training of the AI.

\section{Conclusions}

ObsIdentify is a promising tool to improve the processing of biodiversity data acquired by citizen scientists. The app has an intuitive layout and provides additional motivations for the user such as achievements, user statistics and challenges, e.g., "upload as many observations of pollinators within a month", encouraging especially younger people to use the app regularly. Most importantly, the quality of data is superior to similar apps and databases, as the validation process is managed only by experts.

The list of potential applications of the growing database is long: estimates on population sizes and rarity can be made, long outdated distribution maps can be updated, phenology can be better evaluated, new insights into interspecific associations can be gained (Schermer \& Hogeweg 2018), collections can be digitized (Hedrick et al. 2020) and openly accessed online, and neobiota and mass die-offs could be detected quicker and respective measures could be taken in time.

With an app called SnailSnap, that was also linked to the database of observation.org, Kerstes et al. (2019) found interesting clues on urban evolution in Cepaea nemoralis thanks to the participation of citizen scientists. Such findings might be made more frequently if a wide audience uses the Obs-Services on a regular basis. "Challenges" or "bounty boards", e.g., "photograph as many specimens of species XY" might get more people involved and could funnel the focus of data input for specific research projects. Those challenges already exist in some form in ObsIdentidfy but are easy to overlook and should be more promoted and used more frequently. This might get users interested and specialized in a more specific taxonomic group, that was featured in a challenge, and maybe lead to data of higher quality.

So far, for beetles there is still room for improvement in species identification accuracy and the number of validators. Usually though, lay people tend to photograph species that are easy to find, and which are also often not hard to identify and thus can be validated by pictures. Results for such species were mostly correct, even if only photographs of "average" quality from only one perspective were used. Nevertheless, providing multiple pictures from different angles can help in the validation process. Unvalidated submissions and observations uploaded with an incorrect name are not worthless, as they can still be corrected/validated retrospectively, although chances for this lower over time. Records with photographs submitted through the ObsIdentify and ObsMapp apps or the related website https://observation.org/ are publicly accessible, making the data also useful to professionals. Experts who stumble upon an erroneous observation can cross-check the associated photographs of any record and report wrong identifications to validators.

For experienced users, there are options to upload and add more details to an existing observation by using the website or the app ObsMapp. This leads to a higher value of observations, as biodiversity data gathered by citizen scientists are often too undetailed to perform comprehensive analysis and to make well-founded predictions or conclusions (Van Eupen et al. 2021). It would be worth considering implementing these additional options in ObsIdentify while keeping the layout user-friendly, like an optional "advanced mode".

So far, the species suggestions work most reliably in the Netherlands, Belgium and Germany. Users can upload observations from other regions as well, but the image recognition will not be as reliable, and the validation is currently either slow or impossible due to the lack of specialized validators. E.g., uploaded pictures of various Lucanidae from Southeast Asia always resulted in the identification (mostly with $100 \%$ confidence) of Lucanus cervus, which does not occur there. This could be countered by tuning the species suggestions so that only native species for a country are suggested. This again would need many more specialized validators for the respective regions who can work on the lists.

Before precise species identifications for groups with many cryptic species, e.g. Staphylinidae, will become possible in the field, experts must first contribute with their own data and help with validation for the respective groups. With enough high-quality data input and validation from experts, results could be improved to a point where the AI might identify cryptic species more confidently than the human observer, as the algorithm might find minute morphological distinct characteristics that are overseen by humans (Hedrick et al. 2020). This can save time and money in biodiversity assessments, as the long and hardy identification through keys could become obsolete. This, however, will take a lot of time and the databases for such commonly overlooked taxa will have to be founded on data from the respective experts and institutions rather than on the occasional observations of users. In turn, users can then contribute observations that have higher rates of correct identification through the algorithm. 
When more validators for certain taxa and regions join this system and the image recognition software is further trained, the Obs-Services yield the potential to become the global tools of choice for biodiversity-related citizen science data gathering. They could be a huge enrichment in the scientific community, as data which are very hard and lengthy to gather as an individual become quickly accessible on a huge scale. If more museums, conservation centres, NGOs, national parks, or governmental institutions would use these tools and assign their own validators for their respective regions, the knowledge on biodiversity could be significantly improved, which is vital to initiate effective conservation efforts. Also, the app should be promoted more in online advertisements, newspapers, and other media so that more potential users and partners are reached.

\section{Acknowledgements}

I would like to thank Prof. Dr. Hendrik Freitag from the Ateneo de Manila University, Philippines and Editor-in-Chief of the Tijdschrift voor Entomologie, for giving me the opportunity to publish this paper as invited article, giving input on the subject and his hospitality during the excursions. I also want to thank Ulrich Haese (Stolberg) who introduced me to the Obs-Services and David Tempelman (Observation International), who provided important information on the functionality of the tools used in this paper and guided me on how to perform validation and taxonomy work on observation.org. Further, I want to thank Thorsten Klumb (Alsdorf), who often accompanies me in the field and is also an active user of the ObsServices. Finally, I thank all proofreaders (Elena Recker (RWTH University Aachen), André Dümont (Alsdorf), Hendrik Freitag and David Tempelman). I sincerely thank Prof. Dr. Menno Schilthuizen (Leiden) and an anonymous reviewer for their comments and suggestions and all those who provided literature.

If you are an expert on a specific species group with proof for your expertise, or an interested institution, no matter where you are located, and consider actively volunteering as a validator, taxonomist, or institutional partner, please do not hesitate to contactinfo@observation.org.

\section{Supplement 1.}

Top in-app results and validation status of submitted observations sorted by family of correct species. This supplement can be accessed at doi: 10.6084/ m9.figshare. 16866181 .

\section{References}

Beijbom, O., P.J. Edmunds, C. Roelfsema, J. Smith, D.I. Kline, B.P. Neal, M J. Dunlap, V. Moriarty, T.-Y. Fan,
C.-J. Tan, S. Chan, T. Treibitz, A. Gamst, B.G. Mitchell \& D. Kriegman, 2015. Towards automated annotation of benthic survey images: variability of human experts and operational modes of automation. - PLoS ONE 10: e0130312. doi: 10.1371/journal. pone.0130312.

Engel, M. S., L.M.P. Ceríaco, G.M. Daniel, P.M. Dellapé, I. Löbl, M. Marinov, R.E. Reis, M.T. Young, A. Dubois, I. Agarwal, P.A. Lehmann, M. Alvarado, N. Alvarez, F. Andreone, K. Araujo-Vieira, J.S. Ascher, D. Baêta, D. Baldo, S.A. Bandeira, P. Barden, D.A. Barrasso, L. Bendifallah, F.A. Bockmann, W. Böhme, A. Borkent, C.R.F. Brandão, S. Busack, S.M. Bybee, A. Channing, S. Chatzimanolis, M.J.M. Christenhusz, J.V. Crisci, G. D’Elía, L.M. da Costa, S.R. Davis, C.A.S. De Lucena, T. Deuve, S.F. Elizalde, J. Faivovich, H. Farooq, A.W. Ferguson, G. Spartaco, F.M. Gonçalves, V. Gonzalez, E. Greenbaum, I.A. HinojosaDíaz, I. Ineich, J. Jiang, S. Kahono, A. Kury, P.H.F. Lucinda, J.D. Lynch, V. Malécot, M.P. Marques, J.W.M. Marris, R.C. McKellar, L.F. Mendes, S. Nihei, K. Nishikawa, A. Ohler, V. Orrico, H. Ota, J. Paiva, D. Parrinha, O.S.G. Pauwels, M.O. Pereyra, L.B. Pestana, P.D.P. Pinheiro, L. Prendini, J. Prokop, C. Rasmussen, M.-O. Rödel, M.T. Rodrigues, S.M. Rodríguez, H. Salatnaya, Í. Sampaio, A. Sánchez-García, M. Shebl, B.S. Santos, M.M. Solórzano Kraemer, A.C. Sousa, P. Stoev, P. Teta, J.-F. Trape, C.V.-D. dos Santos, K. Vasudevan, C.J. Vink, G. Vogel, P. Wagner, T. Wappler, J.L. Ware, S. Wedmann \& C.K. Zacharie, 2021. The taxonomic impediment: a shortage of taxonomists, not the lack of technical approaches. - Zoological Journal of the Linnean Society 193: 381-387. doi: 10.1093/ zoolinnean/zlab072/6374389.

Gomez Villa, A., A. Salazar \& F. Vargas, 2017. Towards automatic wild animal monitoring: Identification of animal species in camera-trap images using very deep convolutional neural networks. Ecological Informatics 41: 24-32. doi: 10.1016/j. ecoinf.2017.07.004.

Hedrick, B.P., J.M. Heberling, E.K. Meineke, K.G. Turner, C.J. Grassa, D.S. Park, J. Kennedy, J.A. Clarke, J.A. Cook, D.C. Blackburn, S.V. Edwards \& C.C. Davis, 2020. Digitization and the future of natural history collections. - BioScience 70: 243-251. doi: 10.1093/ biosci/biz163.

Hochkirch, A., M.J. Samways, J. Gerlach, M. Böhm, P.H. Williams, P. Cardoso, N. Cumberlidge, P.J. Stephenson, M. Seddon, V. Clausnitzer, P. Borges, G.M. Mueller, P. Pearce-Kelly, D.T. Raimondo, A. Danielczak \& K.D. Dijkstra, 2020. A strategy for the next decade to address data deficiency in neglected biodiversity. - Conservation Biology 35: 502-509. doi: 10.1111/cobi.13589.

Kaufmann, P. \& R. Lindner, 2021. Biodiversitätsdaten, Citizen Science und Online-Erfassungssysteme Überblick und Erfahrungsbericht. - Anliegen Natur 43: 93-100.

Kerstes, N.A.G., T. Breeschoten, V.J. Kalkman \& M. Schilthuizen, 2019. Snail shell colour evolution in urban heat islands detected via citizen science. - Communications

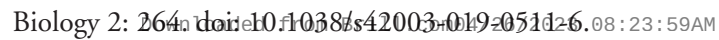
via free access 
Laufer, L., C. Höppner \& A. Nöllert, 2020. 3.9 Gelbbauchunke (Bombina variegata). - Rote-Liste-Gremium Amphibien und Reptilien: Rote Liste und Gesamtartenliste der Amphibien (Amphibia) Deutschlands. Naturschutz und Biologische Vielfalt 170: 42-43. doi: $10.19213 / 972174 /$.

LeCun, Y., Y. Bengio \& G. Hinton, 2015. Deep learning. - Nature 521: 436-444. doi: 10.1038/ nature14539.

Observation International, 2021. Partners. - https://observation.org/pages/partners/.

Oncevay-MarcosA., R. Juarez-Chambi, S. KhlebnikovNúñez \& C. Beltrán-Castañón, 2015. Leaf-based plant identification through morphological characterization in digital images. - In: G. Azzopardi \& N. Petkov (Eds), Computer Analysis of Images and Patterns. CAIP 2015. Lecture Notes in Computer Science, vol. 9257, pp. 326-335. Springer, Cham. doi: 10.1007/978-3-319-23117-4_28.
Roger, E., P. Tegart, R. Dowsett, M.A. Kinsela, M.D. Harley \& G. Ortac, 2020. Maximising the potential for citizen science in New South Wales. - Australian Zoologist 40: 449-461. doi: 10.7882/AZ.2019.023.

Schermer, M. \& L. Hogeweg, 2018. Supporting citizen scientists with automatic species identification using deep learning image recognition models. - Biodiversity Information Science and Standards 2: e25268. doi: 10.3897/biss.2.25268.

Schlüpmann, M., A. Geiger, U. Haese, A. Kronshage, T. Mutz \& D. Specht, 2021. Arbeitskreis Amphibien und Reptilien Nordrhein-Westfalen - Jahresbericht 2020. - Rana 22: 159-167.

Van Eupen, C., D. Maes, M. Herremans, K.R.R. Swinnen, B. Somers \& S. Luca, 2021. The impact of data quality filtering of opportunistic citizen science data on species distribution model performance. - Ecological Modelling 444: 109453. doi: 10.1016/j. ecolmodel.2021.109453. 